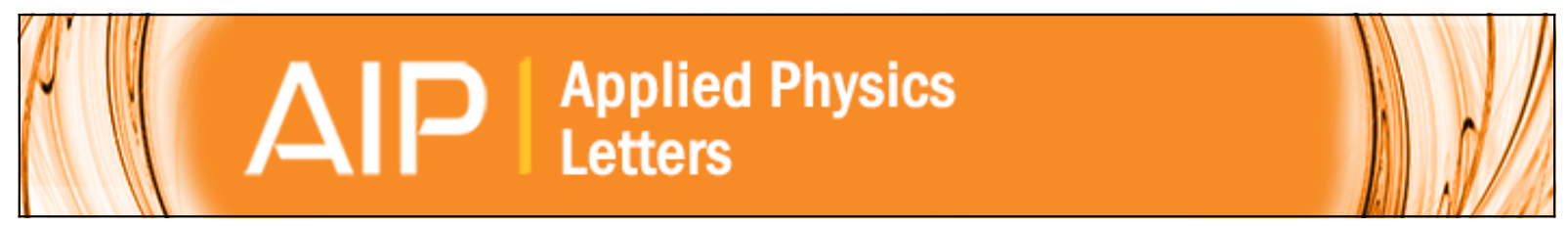

\title{
Current-free double-layer formation in a high-density helicon discharge
}

Christine Charles and Rod Boswell

Citation: Applied Physics Letters 82, 1356 (2003); doi: 10.1063/1.1557319

View online: http://dx.doi.org/10.1063/1.1557319

View Table of Contents: http://scitation.aip.org/content/aip/journal/apl/82/9?ver=pdfcov

Published by the AIP Publishing

\section{Articles you may be interested in}

Evidence of current free double layer in high density helicon discharge

Phys. Plasmas 20, 013510 (2013); 10.1063/1.4789455

Current-free double layers: A review

Phys. Plasmas 18, 122105 (2011); 10.1063/1.3664321

The ion velocity distribution function in a current-free double layer

Phys. Plasmas 12, 093502 (2005); 10.1063/1.2033647

Time development of a current-free double-layer

Phys. Plasmas 11, 3808 (2004); 10.1063/1.1764829

Radio frequency power deposition in a high-density helicon discharge with helical antenna coupling Phys. Plasmas 7, 4308 (2000); 10.1063/1.1288399

\section{Model PS-100}

Tabletop Cryogenic Probe Station

\section{Lake Shore} CRYOTRONICS

\author{
An affordable solution for
} a wide range of research 


\title{
Current-free double-layer formation in a high-density helicon discharge
}

\author{
Christine Charles ${ }^{\mathrm{a})}$ and Rod Boswell \\ Space Plasma and Plasma Processing group, Plasma Research Laboratory, Research School of Physical \\ Sciences and Engineering, The Australian National University, Canberra, ACT 0200 Australia
}

(Received 15 July 2002; accepted 7 January 2003)

\begin{abstract}
A strong, current-free, electric double-layer with $e \Phi / k T_{e} \sim 3$ and a thickness of less than 50 debye lengths has been experimentally observed in an expanding, high-density helicon sustained rf (13.56-MHz) discharge. The rapid potential decrease is associated with the "neck" of the vacuum vessel, where the glass source tube joins the aluminum diffusion chamber, and is only observed when the argon gas pressure is less than about 0.5 mTorr. The upstream electron temperature $T_{e}$ appears $25 \%$ greater than the downstream $T_{e}$, and there is a density hole on the downstream edge. This experiment differs from others in that the potentials are self-consistently generated by the plasma itself, and there is no current flowing through an external circuit. The plasma electrons are heated by the rf fields in the source, provide the power to maintain the double-layer, and hence accelerate ions created in the source out into the diffusion chamber. (C) 2003 American Institute of Physics. [DOI: 10.1063/1.1557319]
\end{abstract}

Electric double layers (DLs) in plasmas have been studied experimentally ${ }^{1-4}$ theoretically, ${ }^{5,6}$ and by computer simulation. $^{7,8}$ The interest has been driven by a desire to explain auroral phenomena observed on spacecraft ${ }^{9-11}$ that are associated with the acceleration of electrons to the earth to produce the visible aurora. The close association of the DLs with ion acoustic solitons ${ }^{12}$ has also been pointed out.

The majority of experiments reporting laboratory DLs generally fall into two types: the first involves creating two separate plasmas at different potentials and allowing them to interact in a central chamber joining the two sources. ${ }^{13,14}$ Stable DLs can be formed by careful manipulation of the external experimental parameters. The other type involves a current flow in cylindrical " $Q$ " machines ${ }^{15}$ or in direct current discharges with an abrupt change in diameter.

An interesting experiment, similar to the one described here, has been reported, in which measurements show a DL in a weakly magnetized system expanding away from a small source. ${ }^{16}$ The authors note that their field-aligned DL contained no trapped or counter-streaming ions and was currentfree. The experiments were carried out in a pulsed system, and the high potential in the upstream of the DL was provided by the anode of the plasma source. However, for pressures above $5 \times 10^{-5}$ Torr, no DLs were observed, and above $4 \times 10^{-4}$ Torr, no energetic ions were observed.

The experiment described in this letter presents a phenomenon of a current-free double-layer in an expanding plasma that could be the basis of an enhanced type of space plasma thruster. It could provide thrust of comparable magnitude to other systems.

A horizontal helicon system consisting of a $15-\mathrm{cm}-$ diameter helicon ${ }^{17}$ source (a 30-cm-long cylindrical glass tube surrounded by a 20 -cm-long double saddle antenna and terminated with a glass plate) is attached contiguously to a

\footnotetext{
a) Also at: the "Département Sciences Pour l'Ingénieur," Centre National de la Recherche Scientifique, France; electronic mail: christine.charles@anu.edu.au
}

32-cm-diam earthed aluminum diffusion chamber [Fig. 1(a)]. The antenna is fed from a rf matching network/generator system operating at $13.56 \mathrm{MHz}$. The argon feed gas is introduced at the top of the source tube, and a turbomolecular/ rotary pumping system is connected to the sidewall of the chamber. The base pressure is a few $10^{-6}$ Torr, the pressure being measured with an ion gauge and a baratron gauge. Two solenoids situated around the source are used to create an expanding magnetic field from the source center to the diffusion chamber. Two field cases are shown in Fig. 1(b): a "high"-field case (solid line) under investigation and a "low"-field case (dotted line) previously studied ${ }^{17}$ and presently used as a reference for calibrating the electric probes. The high-field case corresponds to a field of about $250 \mathrm{G}$ in the source center decreasing to a few tens of gauss in the diffusion chamber.

The end plate of the diffusion chamber is equipped with an axially movable, retarding field energy analyzer (RFEA) and a Langmuir probe (LP). The RFEA measurements are made on-axis with the entrance orifice facing radially; a detailed description and optimization of the analyzer has been previously reported, and showed that the energy resolution is $0.3 \mathrm{eV}$ or better. ${ }^{18}$ Since the various grid voltages used in this experiment are somewhat different from those in Ref. 18, the energy resolution might differ slightly, but is expected to be less than $1 \mathrm{eV}$. Although rf electric fields are very likely present in the helicon source, ${ }^{17,19}$ the range of density is sufficiently low (low end of $10^{10} \mathrm{~cm}^{-3}$ ) at pressures lower than 1 mTorr that the ion transit time through the sheath in front of the earthed analyzer body and first grid is larger than the rf period, and a single peak is observed for all $z$ when plotting the derivative of the measured current versus discriminator voltage characteristic $I\left(V_{d}\right)$. We stress here that we are not measuring the actual ion energy distribution function, but rather the energy of the ions falling through the presheath and sheath of the earthed analyzer. We define the plasma potential as the energy at the maximum derivative in the $I$ 

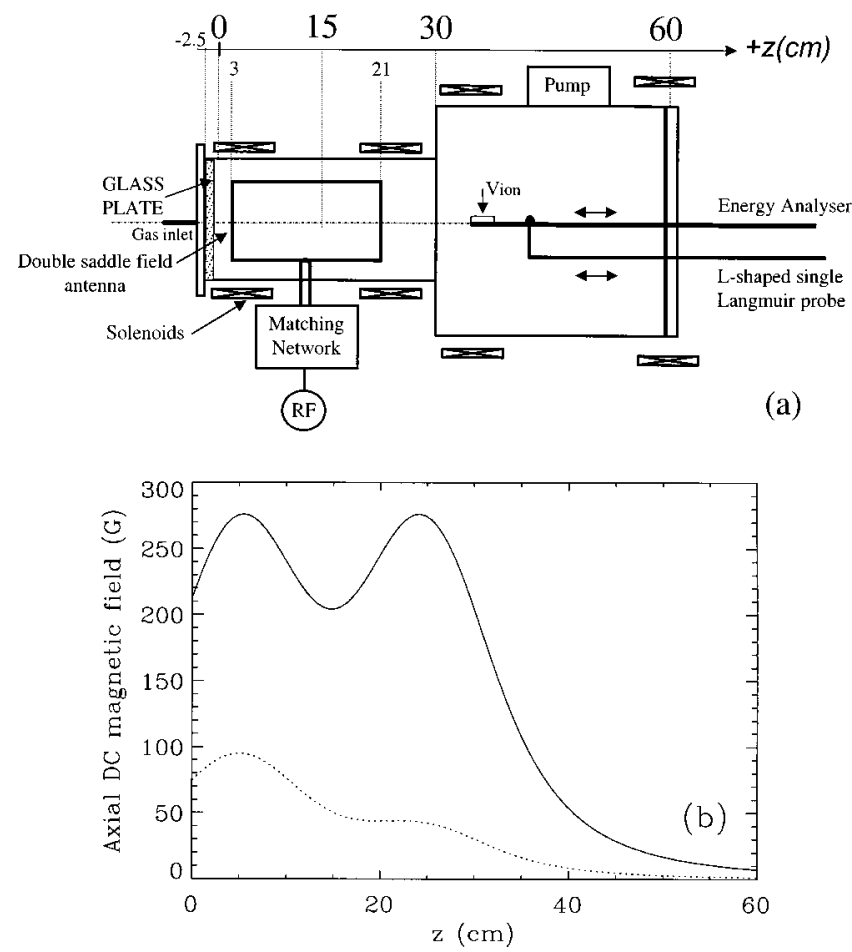

FIG. 1. (a) Schematic of "Chi-Kung," a horizontal helicon system, showing major components and (b) $B_{z}$ component of the dc magnetic field along axis for the high (solid line)- and low (dotted line)- field cases.

versus $V_{d}$ curves. The $I(0)$ current is calibrated in density by using the LP in the low-field case. ${ }^{17}$

Calibration and a check of the reliability of the probes was made using a lower magnetic field configuration [Fig. 1(b)] with higher pressure and power operating conditions. These are conditions we have used previously and which yield plasma parameters that are internally consistent. ${ }^{17}$ The on-axis plasma potential and plasma density are shown in Figs. 2(a) and 2(b), respectively. At 3 mTorr and typically at pressures above $1 \mathrm{mTorr}$, the plasma is created in the source and expands into the diffusion chamber, with the decrease in the density being accompanied by a decrease in the plasma potential, which follows the Boltzmann relation for electrons in the diffusion chamber: ${ }^{20,21}$

$$
n=n_{0} \exp \left(-e \Phi / k T_{e}\right) .
$$

The slope of the natural logarithm of the density against plasma potential yields a temperature of $4.9 \mathrm{eV}$, which is within $0.5 \mathrm{eV}$ of the $4.4 \mathrm{eV}$ derived from the LP. From this we can gain confidence that both diagnostics are operating properly for these pressures and that the plasma is expanding in an understandable manner. The cross section for charge exchange for low-energy argon ions is about 5 $\times 10^{-15} \mathrm{~cm}^{2}$, which results in a mean free path (mfp) of 2 $\mathrm{cm}$ at 3 mTorr. With the measured field of $50 \mathrm{~V} \mathrm{~m}^{-1}$ in the chamber, the ions would only acquire about $1 \mathrm{eV}$ of energy before being scattered, and one would expect a fairly isotropic (if nonthermal) distribution of the ion energies. The RFEA, which is oriented to measure ions in the radial direction, would measure an ion current that could be correctly interpreted as a good measure of the plasma density.

The plasma potential and plasma density obtained with the RFEA aiong the $z$-axis of the reactor are shown in Figs.
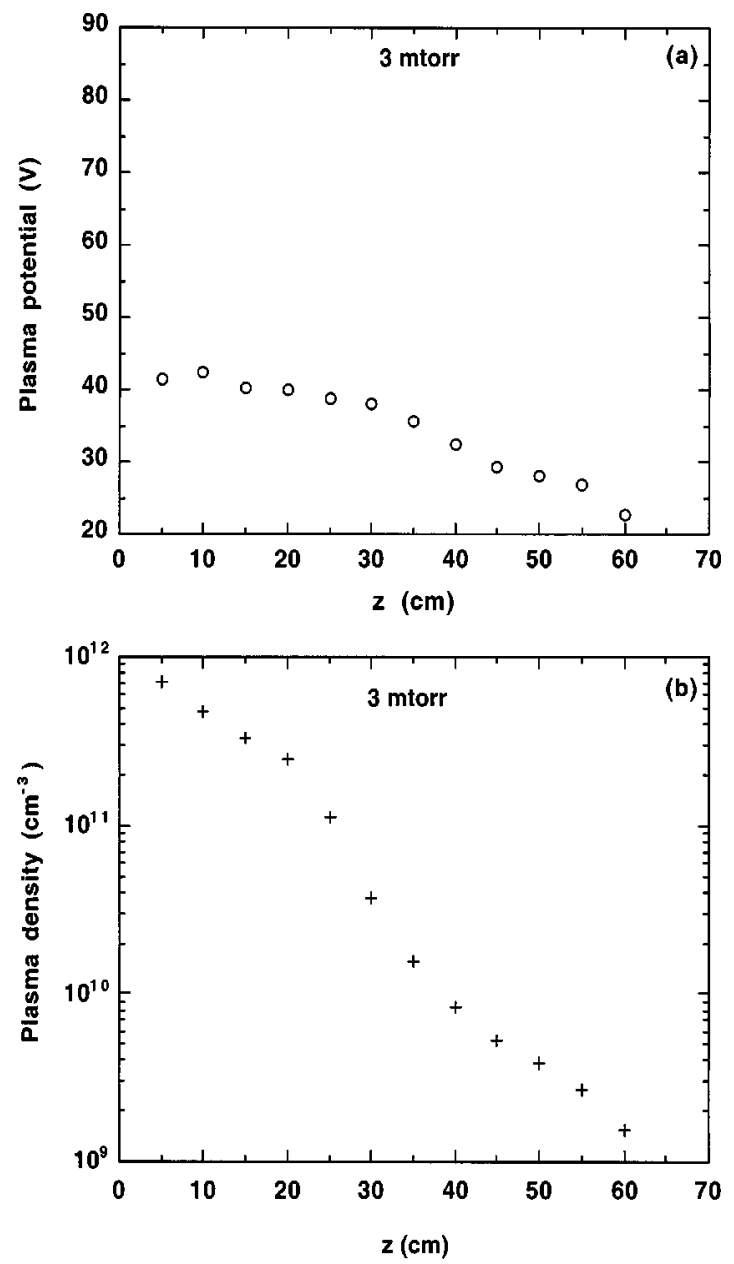

FIG. 2. (a) Plasma potential and (b) plasma density measured with the energy analyzer along the $z$-axis for 3-mTorr pressure, $800 \mathrm{~W}$ rf power and low-field [Fig. 1(b)] conditions, respectively.

3(a) and 3(b), respectively for the high-field, low-pressure (0.2-mTorr) case. The measurements are made during one run for increasing values of $z$, starting in the source center.

There is a rapid and discontinuous change in the plasma potential close to the exit of the source, accompanied by a dip in the plasma density. In the source, the plasma potential drops from around $80 \mathrm{~V}$ in the center $(z=+15 \mathrm{~cm})$ to about $57 \mathrm{~V}$ at the top of the DL at $25 \mathrm{~cm}$, which is just inside the source. The results of Fig. 3(a) show that the field in the source is reasonably constant along the axis and is equal to about $220 \mathrm{~V} \mathrm{~m}^{-1}$. Given that the $\mathrm{mfp}$ for charge exchange is about $30 \mathrm{~cm}$ at 0.2 mTorr, we would expect a large proportion of ions to be accelerated to at least $20 \mathrm{eV}$, which is highly supersonic. The electron temperature determined from the $\mathrm{LP}$ is measured to be $8 \pm 0.5 \mathrm{eV}$ in the diffusion chamber, which is what would be expected at this low pressure. In the source, the fit of the electron current gives an electron temperature of $10 \pm 0.5 \mathrm{eV}$. Assuming that $V_{p}-V_{f}$ is approximately $5.2 T_{e}$ for an argon plasma, the plasma $(72.8-\mathrm{V})$ and floating $(21.5-\mathrm{V})$ potentials measured with the LP positioned at $z=20 \mathrm{~cm}$ yield an electron temperature of $9.9 \mathrm{eV}$. The electron temperature upstream of the DL is $25 \%$ greater than downstream, in agreement with previous reports. ${ }^{20}$ Other authors have also noted the existence of a density dip on the low-potential side of the DL. ${ }^{16,22}$

The interpretation of RFEA measurements can be diffi- 

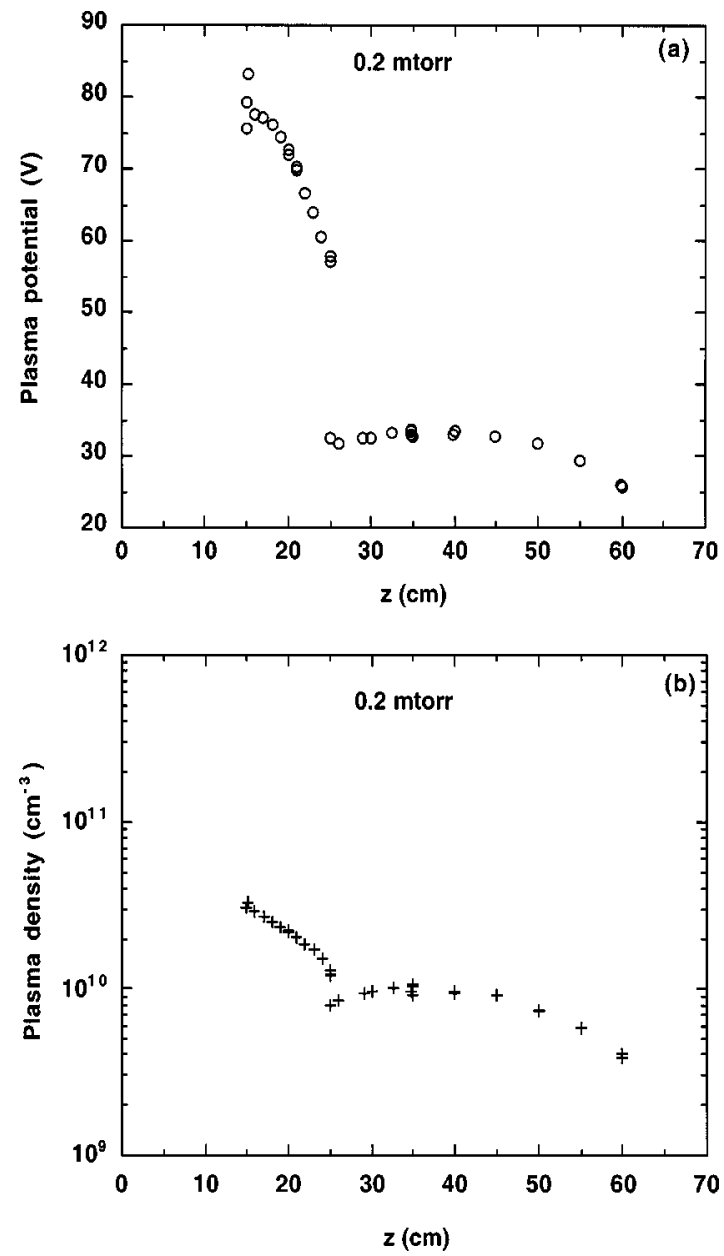

FIG. 3. (a) Plasma potential and (b) plasma density measured with the energy analyzer along the $z$-axis for 0.2 -mTorr pressure, $250 \mathrm{~W}$ rf power and high field [Fig. 1(b)] conditions, respectively.

cult in a flowing plasma, especially in the present case, in which the flow is very supersonic. Consequently, the radially oriented RFEA would underestimate the plasma density, since it would not collect all the ions accelerated by the electric field of the expanding plasma. However, it should measure the local plasma potential accurately, with the width of the derivative reflecting collisional processes in the presheath and some broadening due to rf oscillations of the sheath.

Although precise density measurements were not possible on axis for the DL condition (low pressure, higher field), our potential measurements are sufficient to characterize the type of DL as current-free, with $e \Phi / k T_{e} \sim 3$, where we use the potential drop of $25 \mathrm{~V}$ at the source exit and the downstream electron temperature ${ }^{13,22}$ of $8 \mathrm{eV}$. Although measurements in the DL itself were difficult due to the probe perturbing the potential structure, just upstream or just downstream, the DL was stable, and the results show that the DL thickness is less than $1 \mathrm{~cm}$, about 50 debye lengths for a plasma density of $1 \times 10^{10} \mathrm{~cm}^{-3}$, making this a narrow DL compared to those measured by other experiments. ${ }^{12,22,23}$ Why the simple expansion of the plasma with its associated axial electric field changes to a DL at low pressure is not yet clear. The position of the DL is close to the source to cham- ber change in diameter, but it is also positioned at the greatest value of the magnetic field. No theoretical analysis is available in the literature and there have been no previous measurements reported. Considerable work in both areas needs to be carried out to explain these results.

In application to spacecraft, the thrust generated by this system, which is defined as the rate of change of momentum, can simply be calculated from the plasma density and the potential drop (including the source), along with an estimate of the ion energy gain before charge exchange. For the present configuration, we estimate a thrust of $2.5 \mathrm{mN}$, which is limited by the gas flow (and therefore the pump) and the power being put into the plasma. In space, the exit of the source can be regarded as a black hole that would increase the gas flow by a factor of around 100. The plasma density in these sources generally increases linearly with the power, so it is reasonable to expect that densities of over $10^{12} \mathrm{~cm}^{-3}$ could be generated. Hence, it could be reasonably expected that this simple configuration could be developed to be a plasma thruster operating in space conditions with a thrust of some hundreds of $\mathrm{mN}$. It is also worth remarking that this phenomenon could be of use in the surface processing industry, where a flux of energetic ions that is uniform over a large area could be of some use. We are pursuing this higher power system with RFEA measurements viewed both along and across the plasma flow.

${ }^{1}$ C. Chan, N. Hershkowitz, A. Ferreira, T. Intrator, B. Nelson, and K. Lonngren, Phys. Fluids 27, 226 (1984).

${ }^{2}$ S. Torven and L. Lindberg, J. Phys. D 13, 2285 (1980).

${ }^{3}$ N. Sato, R. Hatekeyama, S. Iizuka, T. Mieno, K. Saeki, J. J. Rasmussen, P. Michelsen, and R. Schrittwieser, Phys. Rev. Lett. 46, 1330 (1981).

${ }^{4}$ M. Guyot and Hollenstein, Phys. Fluids 26, 1596 (1983).

${ }^{5}$ S. Izuka, K. Saeki, N. Sato, and Y. Hatta, Phys. Rev. Lett. 43, 1404 (1979).

${ }^{6}$ S. Torven, Phys. Rev. Lett. 47, 1053 (1981).

${ }^{7}$ G. Knorr and C. K. Goertz, Astrophys. Space Sci. 31, 209 (1974).

${ }^{8}$ M. K. Hudson, W. Lottko, I. Roth, and E. Witt, J. Geophys. Res., 88, 916 (1983).

${ }^{9}$ M. Temerin, M. H. Boehm, and F. S. Mozer, Geophys. Res. Lett. 8, 799 (1981).

${ }^{10}$ M. Temerin, K. Cerny, W. Lotko, and F. S. Mozer, Phys. Rev. Lett. 48, 1175 (1982)

${ }^{11}$ R. E. Ergun, Y. J. Su, L. Anderson, C. W. Carlson, J. P. McFadden, F. S. Mozer, D. L. Newman, M. V. Goldman, and R. J. Strangeway, Phys. Rev. Lett. 87, 045003 (2001).

${ }^{12}$ T. Sato and H. Okuda, Phys. Rev. Lett. 44, 740 (1980).

${ }^{13}$ P. Coakley and N. Hershkowitz, Phys. Fluids 22, 1171 (1979).

${ }^{14}$ C. Hollenstein, M. Guyot, and E. S. Weibel, Phys. Rev. Lett. 45, 2110 (1980).

${ }^{15}$ S. Iizuka, P. Michelsen, J. Juul Rasmussen, R. Schrittwieser, R. Hatakeyama, K. Saeki, and N. Sato, Phys. Rev. Lett. 48, 145 (1982).

${ }^{16}$ G. Hairapetian and R. L. Stenzel, Phys. Fluids B 3, 899 (1991).

${ }^{17}$ C. Charles, A. W. Degeling, T. E. Sheridan, J. H. Harris, M. A. Lieberman, and R. W. Boswell, Phys. Plasmas 7, 5232 (2000).

${ }^{18}$ G. D. Conway, A. J. Perry, and R. W. Boswell, Plasma Sources Sci. Technol. 7, 337 (1998).

${ }^{19}$ A. J. Perry, G. D. Conway, R. W. Boswell, and H. Persing, Phys. Plasmas 9, 3171 (2002).

${ }^{20}$ C. Chan, N. Hershkowitz, and K. E. Lonngren, Phys. Fluids 26, 158 (1983).

${ }^{21}$ C. Charles, R. W. Boswell, and R. K. Porteous, J. Vac. Sci. Technol. A 10, 398 (1992).

${ }^{22}$ D. L. Newman, M. V. Goldman, R. E. Ergun, and A. Mangeney, Phys. Rev. Lett. 87, 255001 (2001).

${ }^{23}$ C. Chan, M. H. Cho, N. Hershkowitz, and T. Intrator, Phys. Rev. Lett. 52, 1782 (1984). 